\title{
Leber und Milz richtig palpieren
}

\author{
Die LEBERPALPATION dient der Beurteilung des Leberrandes und der Ein- \\ schätzung der Lebergröße. Die Milzpalpation dient der Beurteilung \\ der Größe des Organs.
}

Palpation von der linken Körperseite des Patienten aus Es gibt verschiedene Möglichkeiten, die Leber zu palpieren. Der Patient liegt dabei jeweils auf einer Untersuchungsliege auf dem Rücken, seine Beine sind zur Entlastung der Wirbelsäule leicht angewinkelt (mit einem Polster unterstützen).

Der Untersucher steht an der linken Körperseite des Patienten etwa auf Höhe seines Beckens. Er schaut in Richtung des Kopfes des Patienten (nach kranial). Seine Palpationshand, wahlweise die rechte oder linke Hand, legt der Untersucher unterhalb des rechten Rippenbogens auf. Seine andere Hand, die sogenannte Führungshand, legt er darüber. Sie sollte etwas wei- ter unterhalb des Rippenbogens aufgelegt werden, sodass etwas „Hautreserve“ entsteht. Erhöhte Hautspannung lässt sich so vermeiden. Es ist auch möglich, bimanuell zu palpieren, dann liegen beide Hände nah nebeneinander.

Als nächstes bittet der Untersucher den Patienten, normal einzuatmen, seine Hände bleiben dabei unbewegt liegen. Danach bittet er den Patienten, langsam und gleichmäßig auszuatmen. Während das Zwerchfell bei der Ausatmung nach oben gleitet, schiebt er die Palpationshand möglichst tief unter den Rippenbogen in Richtung Leber (s.Abb. 1).
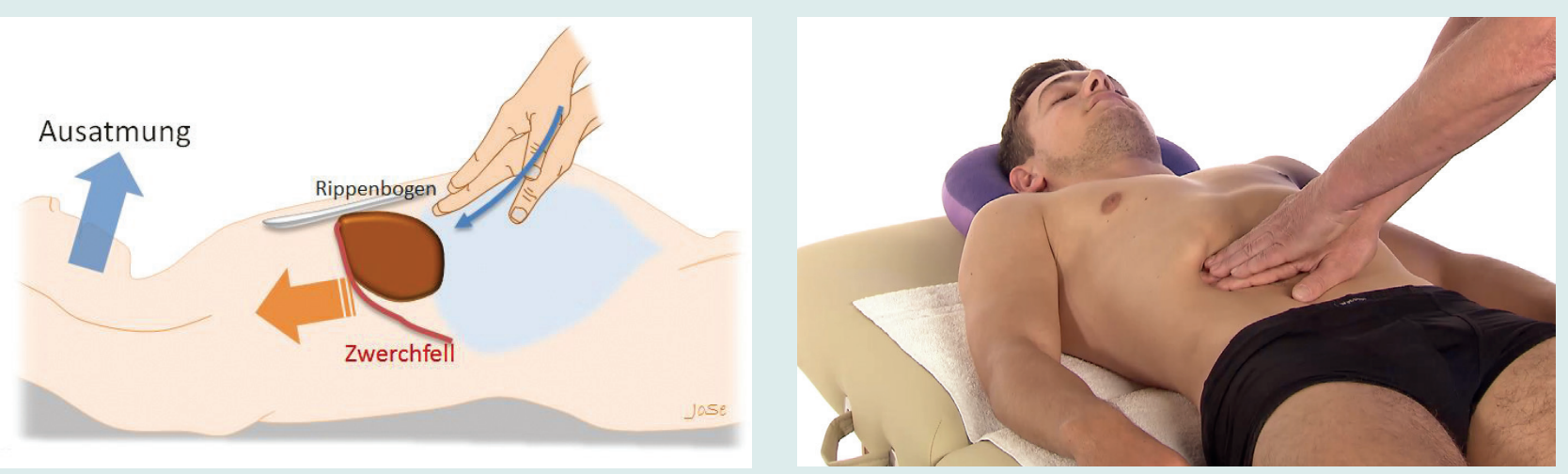

Abb.1 Der Untersuchende schiebt, während der Patient ausatmet, die Palpationshand unter den Rippenbogen in Richtung Leber.
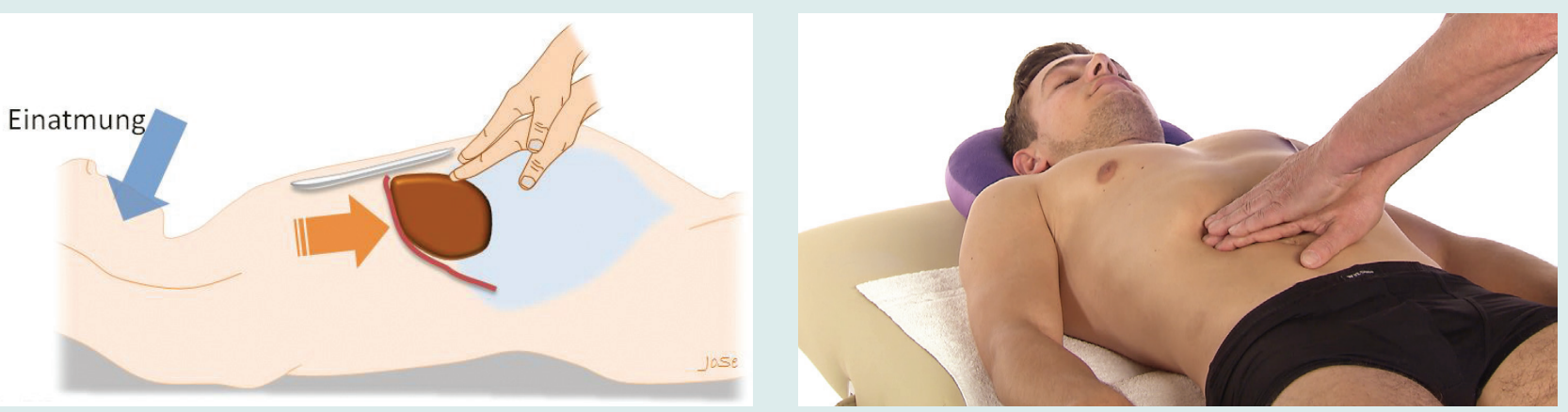

Abb.2 Durch die Einatmung wird das Zwerchfell nach unten gedrückt und die Leber dabei nach unten geschoben. Der Leberrand gleitet unter die Fingerkuppen. 
Danach fordert er den Patienten auf, wieder tief einzuatmen. Die Palpationshand bleibt währenddessen in dieser Position fixiert. Es ist wichtig, dem Druck des Zwerchfells standzuhalten, das sich während der Einatmung nach unten schiebt und dabei auch die Leber nach unten drückt. Der vordere, untere Leberrand gleitet dabei unter die Fingerkuppen des Untersuchers, sodass man ihn nun tasten kann (s.Abb.2). Den Patienten jetzt wieder ausatmen lassen und die Hände zurücknehmen.

\section{Palpation von der rechten Körperseite des Patienten aus}

Die Untersuchung kann auch ausgeführt werden, während der Untersucher an der rechten Körperseite des Patienten steht der Seite also, auf der sich die Leber befindet. Viele Untersuchende empfinden die Untersuchung von dieser Seite aus jedoch als unangenehm, weil die Handgelenke dabei stärker angewinkelt werden müssen und dadurch eventuell nicht ausreichend Kraft aufgebracht werden kann. Die Untersuchung folgt dann weiter dem Vorgehen entsprechend der Palpation von der linken Körperseite des Patienten aus.

\section{Palpation von oben}

Die Leber lässt sich auch von oben palpieren. Dazu steht der Untersuchende auf Schulterhöhe des Patienten an der rechten Körperseite. Die Untersuchung folgt dann weiter dem Vorgehen entsprechend der Palpation von der linken Körperseite des Patienten aus.

\section{Befunde bei der Palpation}

Mit der Leberpalpation können Sie die Konsistenz des unteren Leberrandes ertasten. Als Befunde können vor allem folgende ermittelt werden:

Physiologischer Befund: Die Leber ist nicht oder geringfügig tastbar; sie hat einen mäßig prallen Rand und eine ähnliche Beschaffenheit wie ein Handballen.

Pathologischer Befund: Eine Fettleber fühlt sich weich und teigig an. Eine Hepatomegalie, beispielsweise bei einer Stauungsleber oder bei Hepatitis, ist gut zu palpieren, fühlt sich prall und elastisch an. Eine höckerige Oberfläche ist die narbige Veränderung bei einer beginnenden oder manifesten Zirrhose.

Rückschlüsse auf die Lebergröße sind nur bedingt aussagekräftig. Verschiedene Quellen beschreiben, dass eine palpable Leber bereits auf einer Vergrößerung basiert. Bei einer physiologischen oder thoraxbedingten Tieflage der gesunden Leber, beispielsweise beim Lungenemphysem, trifft dieses jedoch nicht zu. Die Lebergröße wird über die Kratzauskultation oder eine Perkussion bestimmt.

\section{Palpation der Milz}

Dieselben Palpationstechniken wie für die Leber können an der linken Körperseite des Patienten zur Befundung der Milz angewendet werden. Es kann hilfreich sein, diese Untersuchung in Seitenlage vorzunehmen. Dazu dreht sich der Patient auf die rechte Seite. Physiologisch ist die Milz nicht tastbar. Eine Vergrößerung, also eine Splenomegalie, findet sich beispielsweise bei Mononukleose, Hämolyse, Leukämien oder Milztumoren.

Wie Sie die Leber- und Milzpalpation richtig ausführen, sehen Sie in diesem Film

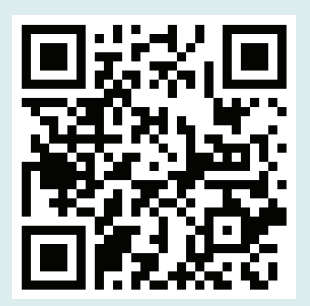

\section{- WEG ZUM FILM}

Mit einem Tablet oder Smartphone können Sie diesen QR-Code einscannen. Sie bekommen dann einen Link angezeigt. Klicken Sie den Link an. Er bringt Sie als Abonnent der DHZ direkt zum Film. Für alle Geräte von Apple finden Sie kostenlose QR-Code-Scanner im AppStore, für Android-Geräte entsprechend bei Google Play. Wenn Sie kein Smartphone besitzen, können Sie den Film aber dennoch ansehen. Geben Sie alternativ die Internetadresse in den Browser Ihres PC ein, die am Ende des Beitrags unter dem Hinweis „Dieser Beitrag ist online zu finden: ..." angegeben ist.

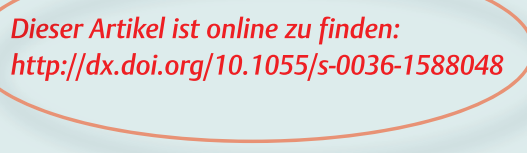

Grafiken: (c) Jürgen Sengebusch Abbildungen: ㄷ Thieme Verlagsgruppe

HP Jürgen Sengebusch, HP Michael Herzog, Senden 